\title{
Subband-Adaptive Shrinkage for Denoising of ECG Signals
}

\author{
S. Poornachandra ${ }^{1}$ and N. Kumaravel ${ }^{2}$ \\ ${ }^{1}$ Department of Biomedical Engineering, SSN College of Engineering, Anna University, Chennai 600025, India \\ ${ }^{2}$ Department of Electronics and Communication Engineering, Anna University, Chennai 600025, India
}

Received 12 March 2005; Revised 8 September 2005; Accepted 28 September 2005

Recommended for Publication by Walter Kellermann

This paper describes subband dependent adaptive shrinkage function that generalizes hard and soft shrinkages proposed by Donoho and Johnstone (1994). The proposed new class of shrinkage function has continuous derivative, which has been simulated and tested with normal and abnormal ECG signals with added standard Gaussian noise using MATLAB. The recovered signal is visually pleasant compared with other existing shrinkage functions. The implication of the proposed shrinkage function in denoising and data compression is discussed.

Copyright (๑) 2006 Hindawi Publishing Corporation. All rights reserved.

\section{INTRODUCTION}

Electrocardiogram (ECG) obtained by noninvasive technique is a harmless, safe, and quick method of cardiovascular diagnosis. The accuracy and content of information extracted from recording require proper characterization of waveform morphologies that needs better preservation of signals and higher attenuation of noise. Recently, wavelet transform has proved to be a useful tool for nonstationary signal analysis. Wavelets provide flexible prototyping environment that comes with fast computational algorithms. A shrinkage method compares empirical wavelet coefficient with a threshold. The coefficient sets it to zero if its magnitude is less than threshold value [1]. The threshold acts as an oracle, which distinguishes between significant and insignificant coefficients. Shrinkage of empirical wavelet coefficients works best when the underlying set of true coefficients of function $f$ is sparse [4].

The wavelet shrinkage was conceptually inspired by the work of Donoho and Johnstone (1995) as well as by the work of Breiman and Bruce and Gao (1996). Donoho et al., developed wavelet shrinkage methods for denoising of function estimation [2]. Among wavelet shrinkage methods, SureShrink is an optimized hybrid scale dependent thresholding scheme based on Stein's unbiased risk estimate (SURE) [5]. It combines universal threshold selection schemes and scale dependent adaptive threshold selection scheme that provide the best estimation results in the sense of $l_{2}$ risk when true function is not known. However, since standard soft shrinkage function is weakly differentiable only in the first order, it does not allow for gradient based optimization method to search for optimal solution for SURE risk [3]. Asymptotically both hard and soft shrinkage estimates are achieved within a factor $\log (n)$ of the ideal performance [1]. The wavelet coefficients at coarsest scale are left intact, while coefficients at all other scales are thresholded via soft shrinkage with universal thresholding

$$
\lambda=\sigma \sqrt{2 \log N}
$$

where $\sigma^{2}$ is the noise variance and $N$ is the length of the signal.

The shrinkage functions proposed by Donoho and Johnstone (1995) are the hard and the soft shrinkage functions:

$$
\begin{aligned}
& \delta_{\lambda}^{H}(x)= \begin{cases}0, & |x| \leq \lambda, \\
x, & |x|>\lambda,\end{cases} \\
& \delta_{\lambda}^{S}(x)= \begin{cases}0, & |x| \leq \lambda, \\
x-\lambda, & x>\lambda, \\
x+\lambda, & x<-\lambda,\end{cases}
\end{aligned}
$$

where $\lambda \in[0, \infty]$ is the threshold. 


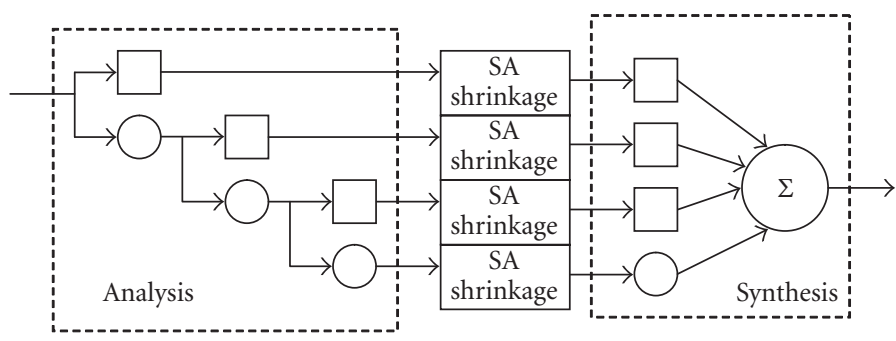

FIGURE 1: Subband-adaptive shrinkage model.

Note that the derivation of standard soft shrinkage function is not continuous. Both hard and soft shrinkages have advantages and disadvantages. The soft shrinkage estimate tends to have bigger bias, due to shrinkage of large coefficients. Due to discontinuities of shrinkage function, hard shrinkage estimate tends to have bigger variance and can be unstable, that is, sensitive to small changes in data [4].

The nonnegative garrote shrinkage functions provides a good compromise between hard and soft shrinkage functions [4] and is first introduced by Breiman (1995),

$$
\delta_{\lambda}^{G}(x)=x\left[1-\left(\frac{\lambda}{x}\right)^{2}\right]_{+}= \begin{cases}0, & |x| \leq \lambda, \\ x-\left(\frac{\lambda^{2}}{x}\right), & |x|>\lambda .\end{cases}
$$

The nonnegative garrote shrinkage function is continuous and approaches identity line as $|x|$ gets large. Breiman applied Garrote shrinkage technique to subset regression to overcome drawbacks of stepwise model selection (equivalent to hard shrinkage in current situations) and ridge regression.

Gao and Bruce (1997) introduced firm shrinkage rule $\delta_{\lambda_{1}, \lambda_{2}}(x)$ :

$$
\delta_{\lambda_{1}, \lambda_{2}}(x)= \begin{cases}0 & \text { if }|x| \leq \lambda_{1}, \\ \operatorname{sgn}(x)\left[\frac{\lambda_{2}\left(|x|-\lambda_{1}\right)}{\left(\lambda_{2}-\lambda_{1}\right)}\right] & \text { if } \lambda_{1}<|x| \leq \lambda_{2}, \\ x & \text { if }|x|>\lambda_{2} .\end{cases}
$$

Though firm shrinkage [6] takes all functional advantages from hard and soft without drawbacks of either, it requires two thresholds. This complicates threshold selection problems further and is computationally expensive for procedures like SURE.

Hyper shrinkage is an optimized thresholding scheme based on universal threshold [1]. The major advantage of $h y$ per shrinkage is nonlinearity; wherein wavelet domain tends to keep a few larger coefficients representing the function while noise coefficients tend to be reduced to zero.

Poornachandra and Kumaravel (2004) proposed hyper shrinkage $\delta_{\lambda}^{\text {hyp }}(x)[7]$,

$$
\delta_{\lambda}^{\text {hyp }}(x)=\tanh \left(\rho^{*} x\right)(|x|-\lambda)_{+}= \begin{cases}0, & |x| \leq \lambda, \\ \tanh \left(\rho^{*} x\right), & |x|>\lambda,\end{cases}
$$

$\rho$ is the boundary contraction parameter, which depends on boundary attaining parameter $\Delta, 10>\Delta>1$, used to retain the exponent behavior of shrinkage function outside redundant area of distribution curve as shown in Figure 1. The value for boundary attaining parameter is purely based on the outcome of replicated trials. In our simulation, we assumed $\Delta=5$. It is observed that for $\Delta<5$, the convergence of the function is poor resulting in loss of stability in output signal and for $\Delta>5$, sets saturated, that is, further increase in the value of $\Delta$ results in the fractional change in the SNR value,

$$
\rho=\frac{\Delta}{\max |x|} .
$$

This paper proposes a novel subband-adaptive shrinkage function that deploys a subband dependent shrinkage scheme based on redundant detection mechanism. While still using a simple shrinkage operation, the proposed model yields superior results in terms of denoising ECG signal.

\subsection{Introduction to wavelet transform}

The special structure of wavelet bases may be appreciated by considering generation of an orthonormal wavelet basis for function $g \in \ell^{2}(\Re)$ (the space of square integrable real functions). The approach of Daubechies (1992) is the most often adopted in applications of wavelets in statistics, mutually orthonormal, functions or parent wavelets: the scaling function, $\varphi$ (sometimes referred to as the father wavelet), and the mother wavelet, $\psi$. Other wavelets in the basis are then generated by translation of scaling function $\varphi$, and dilations and translations of mother wavelet $\psi$ using the relationships

$$
\begin{gathered}
\varphi_{j_{0} k}(t)=2^{j_{0} / 2} \varphi\left(2^{j_{0}} t-k\right), \\
\psi_{j k}(t)=2^{j / 2} \psi\left(2^{j} t-k\right), \\
j=j_{0}, j_{0}+1, \ldots ; k \in Z
\end{gathered}
$$

for some fixed $j_{0} \in \mathbb{Z}$, where $\mathbb{Z}$ is set of integers. The $2^{j / 2}$ term maintains unity norm of the basis function at various scales and $j$ and $k$ are the scaling and translation parameters, respectively. A unit increase in $j$ in (7) has no effect on scaling function $\left(\varphi_{j_{0} k}\right.$ has a fixed width), but packs oscillations 
of $\psi_{j k}$ into half the width (doubles its scale or resolution). A unit increase in $k$ in (7) shifts the location of both $\varphi_{j_{0} k}$ and $\psi_{j k}$, the former by a fixed amount $\left(2^{-j_{0}}\right)$ and the latter by an amount proportional to its width $\left(2^{-j}\right)$. Given the wavelet basis, a function $g \in \ell^{2}(\mathfrak{R})$ is then represented in a corresponding wavelet series as

$$
g(t)=\sum_{k \in Z} c_{j_{0} k} \varphi_{j_{0} k}(t)+\sum_{j=j_{0}}^{\infty} \sum_{k \in Z} w_{j k} \psi_{j k}(t),
$$

with $c_{j_{0} k}=\left\langle g, \varphi_{j_{0} k}\right\rangle$ and $w_{j k}=\left\langle g, \psi_{j k}\right\rangle$ (where $\langle\cdot, \cdot\rangle$ is the standard $\ell^{2}$-inner product of two functions: $\left\langle g_{1}, g_{2}\right\rangle=$ $\left.\int_{R} g_{1}(t) g_{2}(t) d t\right)$.

The wavelet expansion (8) represents the function $\mathbf{g}$ as a series of successive approximations.

Given a vector of function value $\mathbf{g}=\left[g\left(t_{1}\right), g\left(t_{2}\right), \ldots\right.$, $\left.g\left(t_{n}\right)\right]^{T}$ of equally spaced points $t_{i}$, the DWT of $\mathbf{g}$ is given by

$$
\mathbf{d}=\mathbf{W g}
$$

where $\mathbf{d}$ is an $n \times 1$ vector comprising both discrete scaling coefficients $u_{j_{0}, k}$ and discrete wavelet coefficients $d_{j, k}$ and $\mathbf{W}$ is an orthogonal $n \times n$ matrix associated with orthonormal wavelet basis chosen. Both $u_{j_{0}, k}$ and $d_{j, k}$ are related to their continuous counterparts $c_{j_{0}, k}$ and $w_{j, k}$ via the relation ships $c_{j_{0}, k} \approx u_{j_{0}, k} / \sqrt{n}$ and $w_{j, k} \approx d_{j, k} / \sqrt{n}$. The factor $\sqrt{n}$ arises because of the difference between continuous and discrete orthonormality conditions. Note that, because of the orthogonality of W, the inverse DWT (IDWT) is simply given by

$$
\mathbf{g}=\mathbf{W}^{\prime} \mathbf{d}
$$

where $\mathbf{W}^{\prime}$ denotes the transpose of $\mathbf{W}$.

This paper is organized as follows: the new shrinkage function is formulated in Section 3, where its implementation is also discussed. Section 3 reports a number of experimental results to demonstrate the performance of the new shrinkage function with other shrinkage functions. Conclusions are drawn in Section 4.

\section{FORMULATIONS AND IMPLEMENTATION}

\subsection{Objective}

The wavelet shrinkage method relies on the basic idea that the energy of a function will often be concentrated in a few coefficients in wavelet domain while the energy of noise is spread among all the coefficients. Therefore, nonlinear shrinkage function in wavelet domain will tend to keep a few larger coefficients representing the function while noise coefficients will tend to be reduced to zero. The conventional wavelet shrinkage methods are proved to be effective in minimum mean square error (MSE) sense.

The main objective of this paper is to reduce the MSE between original ECG $f$ and denoised ECG $\hat{f}$. Assume the observed data vector as

$$
\mathbf{y}=\left[y_{1}, y_{2}, \ldots, y_{N}\right] \in \mathfrak{R}^{N}
$$

at equispaced location $x_{N}$, then

$$
y_{i}=f_{i}+\sigma z_{i}, \quad i=1,2, \ldots, N,
$$

where $f_{i}$ is a deterministic signal and $\left\{z_{i}\right\}$ are Gaussian random variables with independent identically distributed (i.i.d) $N(0, \sigma)$. The goal of this paper is to estimate $f$ with small mean square error (MSE), that is, to find an estimate $\hat{f}$ with small $\ell_{2}$ risk:

$$
R(\hat{f}, f)=\frac{1}{N} \sum_{i=0}^{N-1} E\left\{\hat{f}_{i}-f_{i}\right\}^{2}
$$

WaveShrink achieves the minimax risk over each functional class in a variety of smoothness classes and with respect to a variety of losses, including $\ell_{2}$ risk [1].

\subsection{A new subband-adaptive shrinkage}

A native method of denoising is equivalent to low-pass filtering naturally included in any dyadic wavelet framework. That is, simply discard channels of highest resolution and allow signal in the channel confined to lower frequency. The problem associated with this linear denoising approach is unsuitable, as it does not remove the noise present in the low frequency channel as most of the signals of biomedical origin are of lower frequencies. For any shrinkage scheme to be effective, an essential property is that the magnitude of signal components is larger than that of existing noise (at least most times).

The proposed subband-adaptive shrinkage, a nonlinear model, works on hyperbolic function, which will outperform the stated soft shrinkage depicted in Figure 1. The analysis section depicted in the block diagram responsible for generation of the empirical wavelet coefficients is shrunk at every subband of wavelet decomposition. The synthesis section is responsible for reconstruction of ECG signal.

For hyperbolic function the distribution characteristic of tangent hyperbola resembles fundamental shrinkage distribution among its family. The pointwise distribution of subband-adaptive shrinkage is compared with both hard and soft shrinkages as illustrated in Figure 2. The pointwise distribution of subband-adaptive shrinkage is comparable with soft shrinkage function; hence it retains the same function stability of soft shrinkage model. The soft shrinkage function exhibits antisymmetric linear characteristics; on the contrary, subband-adaptive shrinkage function exhibits antisymmetric exponential characteristics (Figure 2). The exponential distribution tends to keep larger empirical coefficients, which represent signal characteristics, and shrinks the remaining empirical coefficients exponentially towards zero. Due to which the total number of coefficients to represent the characteristics of ECG is retained and hence better signalto-noise ratio (SNR) and data compression are achieved. It can be seen that the proposed subband-adaptive shrinkage function also holds the symmetry as in case of soft shrinkage. This shrinkage distribution of subband-adaptive function gives a profile closer to the form of a minimum MSE estimate of a Laplacian signal in Gaussian noise. 


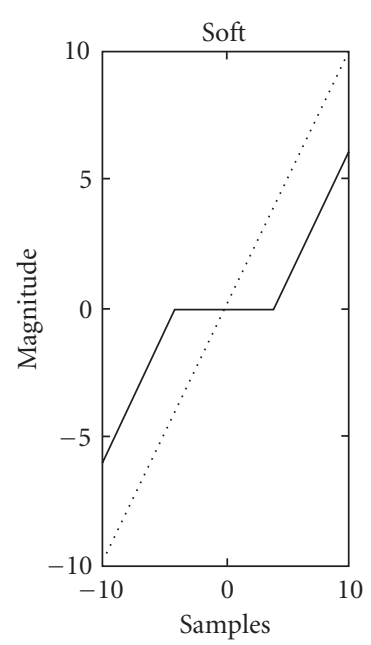

(a)

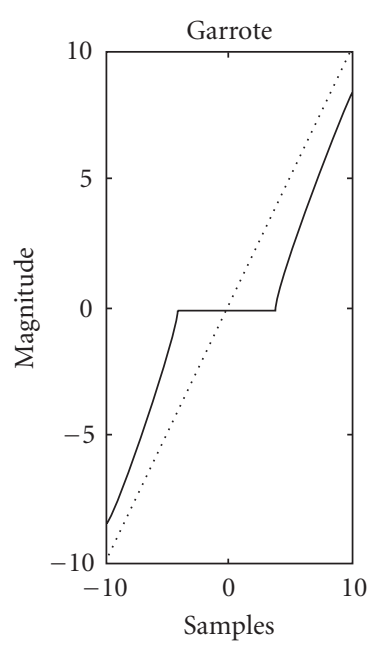

(c)

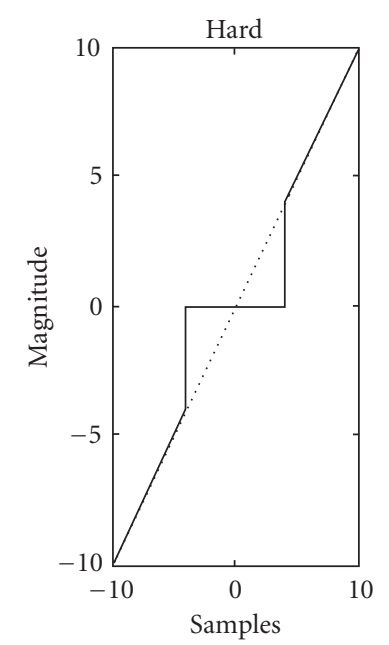

(b)

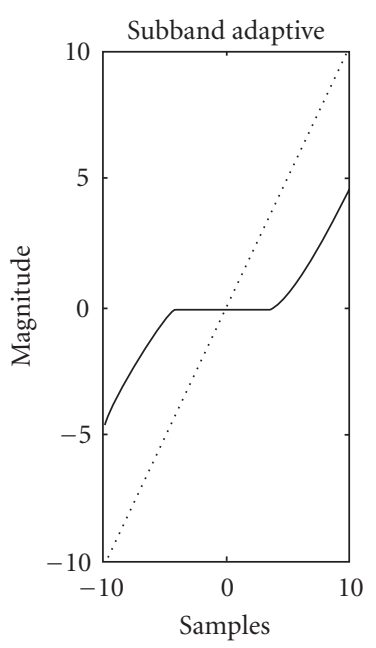

(d)

FIGURE 2: Pointwise distribution of various shrinkage functions while the dotted line represents the original data samples.

The subband-adaptive shrinkage model is expressed as

$$
\delta_{\lambda}^{S A}(x)= \begin{cases}\rho\left[\frac{1-\lambda_{j}^{-2 \lambda_{j} x}}{1+\lambda_{j}^{-2 \lambda_{j} x}}\right], & |x| \geq \lambda_{j}, \\ 0, & |x|<\lambda_{j},\end{cases}
$$

where

$$
\rho=\frac{\Delta}{\max |x|}
$$

$\rho$ is the boundary contraction parameter, which depends on boundary attaining parameter $\Delta, 10>\Delta>1$, used to retain the exponent behavior of shrinkage function outside the redundant area of distribution curve as illustrated in Figure 2. The value for boundary attaining parameter is based on the outcome of replicated trials. It is observed that for $\Delta<5$, convergence of function is very poor resulting in loss of stability in output signal and for $\Delta \gg 5$. Further increase in the value of $\Delta$ results in fractional change in SNR value. In this paper, $\Delta$ is assumed to be 5.1 ing.

The general algorithm for wavelet shrinkage is the follow-

(1) Apply DWT to signal vector $\mathbf{y}$ and obtain empirical wavelet coefficients at scale $j$, where $j=1,2, \ldots, J$.

(2) Apply subband-adaptive shrinkage to empirical wavelet coefficients at each scale $j$.

(3) Estimated wavelet coefficients are obtained based on threshold $\lambda=\left[\lambda_{1}, \lambda_{2}, \ldots, \lambda_{j}\right]^{T}$. Different thresholds are used at different scales.

(4) The estimate of function $\hat{f}$ can be obtained by taking inverse DWT. 


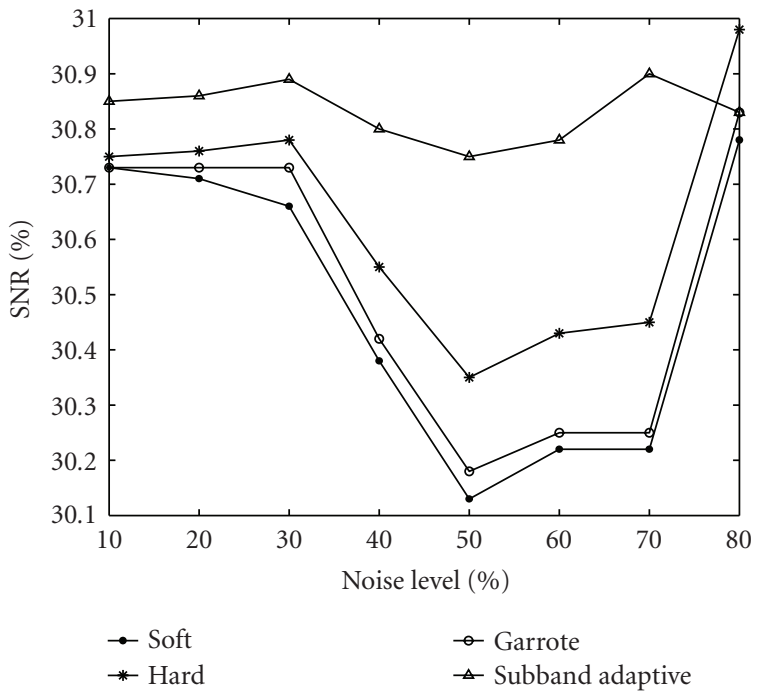

(a)

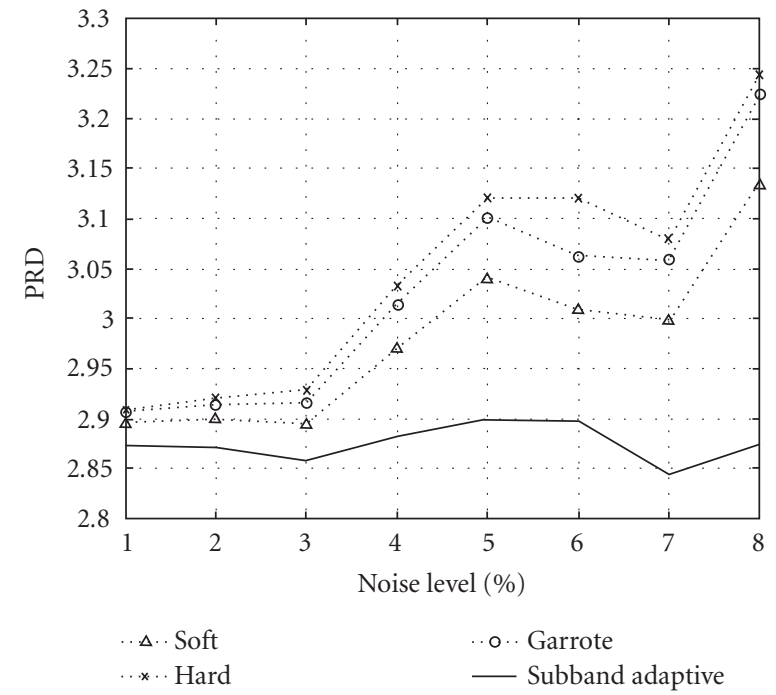

(b)

FIGURE 3: (a) SNR (dB) in the denoised ECG signal versus noise in original ECG signal (b) PRD (\%) in the denoised ECG signal versus noise in the original ECG signal.

\section{RESULTS AND DISCUSSION}

The practical ECGs with 1000 samples are downloaded from the PhysioBank with sampling rate $360 \mathrm{~Hz}$. The simulation was carried out in MATLAB environment. The study was conducted on 50 different ECGs obtained from various limb lead systems of 30 patients. The test was conducted both on normal and abnormal ECGs such as acute myocarditis, right atrial enlargement which results in $\mathrm{P}$ wave and QRS wave abnormalities, right ventricular hypertrophy, and so forth, for the robustness of the proposed shrinkage function.

During simulation various wavelet functions were used for testing denoising of ECG signals such as Daubechies wavelets (DB1 to DB10), Coifman wavelets (COIF1 to COIF8), Meyer wavelet, and Symlet wavelets (SYM1 to SYM8) and found that higher-order functions of all wavelet families produce good denoising effect. Gaussian noise of different standard deviation has been added to the original (noise free) ECG for testing denoising efficiency of the proposed model. It is clear from Figure 3(a) that the SNR performance of other shrinkage models fails to achieve constant SNR value under increased noise condition whereas subband adaptive is consistent.

The SNR is defined as

$$
\mathrm{SNR}(\mathrm{dB})=20 \log \left[\frac{\text { Original ECG }}{\text { Original ECG }- \text { Noisy ECG }}\right] .
$$

The performance of the model for compression has been compared using the percentage root mean square difference (PRD).
PRD is defined as

$$
\operatorname{PRD}=\sqrt{\frac{\sum_{i=1}^{N}\left[x_{\text {original }}(i)-x_{\text {recovered }}(i)\right]^{2}}{\sum_{i=1}^{N}\left[x_{\text {original }}(i)\right]^{2}}} \times 100 \%
$$

where $x_{\text {original }}(i)$ and $x_{\text {recovered }}(i)$ are the $i$ th sample of the original and recovered ECG signals, respectively. Low values of PRD have been obtained for proposed subband-adaptive shrinkage technique. Though the experiment has been conducted on 50 different ECG signals of different origin, Table 1 projects the detailed comparison of SNR (dB) and PRD (\%) for various ECG signals: $\mathrm{m} 105 \mathrm{a}$ is measured using main leadII, m104b is measured at chest lead- $V_{2}$ when the rate of paced rhythm is close to that of the underlying sinus rhythm, resulting in many pacemaker fusion beats and the premature ventricular contractions (PVCs) are multiform. Several bursts of muscle noise occur, but the signals are generally of good quality, m203b is measured from chest lead- $V_{1}$ when the PVCs are multiform, and there are QRS morphology changes in the upper channel due to axis shifts. There is considerable noise in both channels, including muscle artifact and baseline shifts, $\mathrm{m} 213 \mathrm{~b}$ is measured at chest lead- $\mathrm{V}_{1}$ when the PVCs are multiform and usually late-cycle, frequently resulting in fusion PVCs. The morphology of the fusion PVCs varies from almost normal to almost identical to that of the PVCs and $\mathrm{m} 219 \mathrm{~b}$ measured at chest lead- $\mathrm{V}_{1}$; following some conversions from atrial fibrillation to normal sinus rhythm pauses up to 3 seconds in duration. The PVCs are multiform. It is quite interesting to know that the SNR of proposed models are equal to hyper shrinkage when additive noise level 
TABLE 1: SNR and PRD comparison of various ECG signals for different noise levels.

\begin{tabular}{|c|c|c|c|c|}
\hline \multirow{2}{*}{ Noise level (\%) } & \multicolumn{2}{|c|}{ Hyper } & \multicolumn{2}{|c|}{ Subband adaptive } \\
\hline & SNR (dB) & PRD (\%) & SNR (dB) & PRD (\%) \\
\hline 10 & 24.2360 & 6.1404 & 24.2306 & 6.1445 \\
\hline 30 & 24.2540 & 6.1278 & 24.2487 & 6.1315 \\
\hline $\mathrm{m} 105 \mathrm{a}$ & 24.2317 & 6.1435 & 24.2143 & 6.1558 \\
\hline 70 & 24.2195 & 6.1521 & 24.1647 & 6.1911 \\
\hline 90 & 24.1777 & 6.1818 & 24.0934 & 6.2421 \\
\hline 10 & 24.7299 & 5.8010 & 24.7300 & 5.8009 \\
\hline 30 & 24.7453 & 5.7908 & 24.7454 & 5.7907 \\
\hline $\mathrm{m} 104 \mathrm{~b}$ & 24.7197 & 5.8426 & 24.6969 & 5.8231 \\
\hline 70 & 24.7074 & 5.8219 & 24.6645 & 5.8449 \\
\hline 90 & 24.7025 & 5.8374 & 24.6426 & 5.8596 \\
\hline 10 & 24.5652 & 5.9121 & 24.5770 & 5.9040 \\
\hline 30 & 24.5835 & 5.8996 & 24.5922 & 5.8937 \\
\hline $\mathrm{m} 203 \mathrm{~b}$ & 24.5597 & 5.9158 & 24.5549 & 5.9191 \\
\hline 70 & 24.5468 & 5.9246 & 24.5096 & 5.9500 \\
\hline 90 & 24.5446 & 5.9261 & 24.4791 & 5.9710 \\
\hline 10 & 24.6339 & 5.8655 & 24.6537 & 5.8521 \\
\hline 30 & 24.6527 & 5.8528 & 24.6693 & 5.8416 \\
\hline $\mathrm{m} 207 \mathrm{~b}$ & 24.6301 & 5.8680 & 24.6449 & 5.8581 \\
\hline 70 & 24.6114 & 5.8807 & 24.5866 & 5.8975 \\
\hline 90 & 24.5908 & 5.8947 & 24.5416 & 5.9282 \\
\hline 10 & 22.9795 & 7.0991 & 22.9781 & 7.0973 \\
\hline 30 & 22.9994 & 7.0795 & 23.0000 & 7.0794 \\
\hline $\mathrm{m} 213 \mathrm{~b}$ & 22.9807 & 7.0952 & 22.9698 & 7.1041 \\
\hline 70 & 22.9196 & 7.1453 & 22.8737 & 7.1832 \\
\hline 90 & 22.9817 & 7.0944 & 22.9259 & 7.1401 \\
\hline 10 & 23.5058 & 6.6790 & 23.4507 & 6.7215 \\
\hline 30 & 23.5264 & 6.6632 & 23.4734 & 6.7039 \\
\hline $\mathrm{m} 219 \mathrm{~b}$ & 23.4870 & 6.6934 & 23.4060 & 6.7562 \\
\hline 70 & 23.5014 & 6.6823 & 23.4080 & 6.7546 \\
\hline 90 & 23.4574 & 6.7163 & 23.3122 & 6.8296 \\
\hline
\end{tabular}

in ECG signal is increased. It is observed that the proposed subband-adaptive shrinkage function demonstrates good denoising results for both normal and abnormal ECG signals.

In this paper, simulation results of various wavelet functions like COIF5 (Figure 4(d)), Mayer (Figure 4(e)), SYM3 (Figure 4(f)), and DB3 (Figure 4(c)) are illustrated. To visualize the denoising ability of ECG signal, a known percentage of Gaussian noise is added with noise free ECG signal. In this paper, the variance of the original (noise free) ECG is considered as maximum variance level of the noise
(Gaussian) to be added. So experiments were conducted for various noise levels from $0 \%-100 \%$ (noise variance is equal to signal variance). It is evident from Figure 4 that DB3 and COIF5 wavelet functions are highly suitable for ECG analysis, as it preserves the edge information of original ECG and does not over smooth the denoised signal. The visual representation of denoised ECG for various noise levels using proposed subband-adaptive shrinkage function is illustrated in Figure 5. It is found that the signal recovery rate decays, as the noise level in the original ECG signal is more than 50\% 


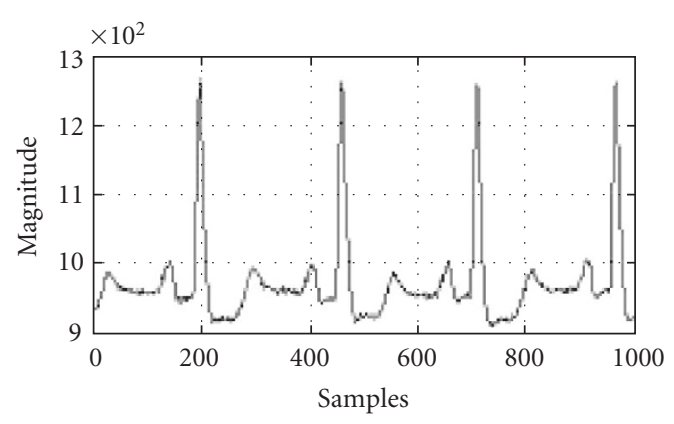

(a)

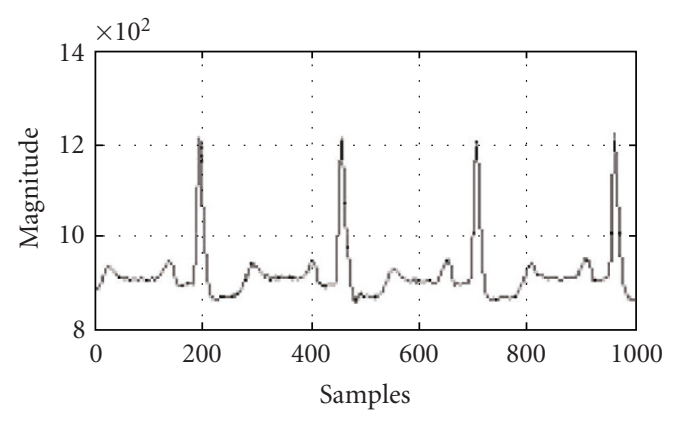

(c)

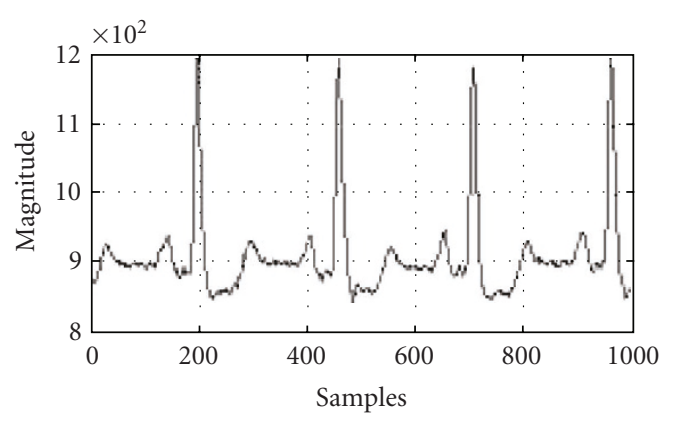

(e)

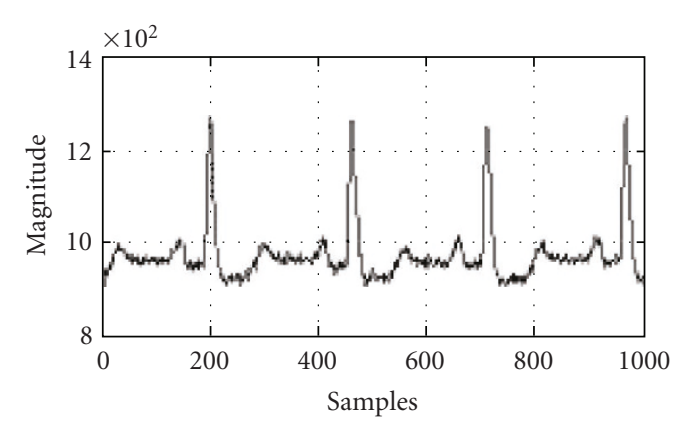

(b)

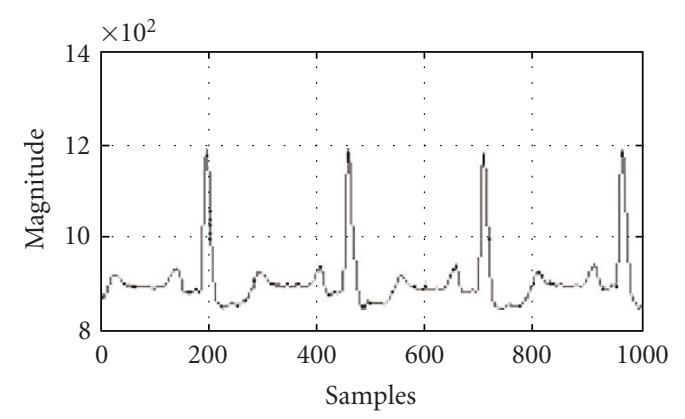

(d)

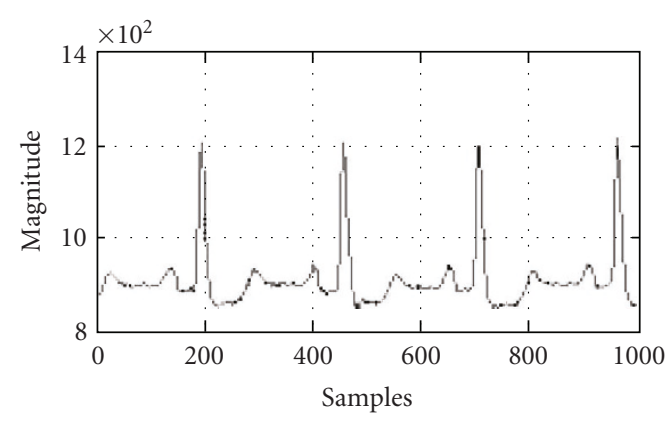

(f)

FIGURE 4: (a) Original ECG signal; (b) noisy ECG (noise level is 50\% of signal variance); recovered ECG (c) using DB3 wavelet; (d) using COIF5 wavelet; (e) using Mayer wavelet; (f) using Symlet wavelet.

of variance of original ECG signal. It is also evident from the results that under high noise conditions, the recovered signal characteristics such as $\mathrm{P}$ wave, QRS complex, and T wave of ECG signal are preserved (Figures $5(\mathrm{~g})$ and $5(\mathrm{j})$ ), that is, the subband-adaptive shrinkage function does not over smooth the ECG signal as in case of hyper shrinkage. The major advantage of subband-adaptive shrinkage function over hyper shrinkage [7] is its signal stability at discontinuities, which makes the subband-adaptive shrinkage unique in its family. The hyper shrinkage exhibits oscillatory behavior at the QRS complex of ECG signal in alternate beats that are almost suppressed in case of subband-adaptive shrinkage.

\section{CONCLUSION}

This paper proposes a novel dynamical nonlinear wavelet domain shrinkage model for signals of cardiovascular origin to reduce noise in signal by shrinking the redundant empirical wavelet coefficients at every subband level. The initial experiment was conducted on a normal ECG and then extended to other abnormal ECG signals. Experiments were conducted by increasing the noise level of both normal and abnormal ECGs and found the proposed subband-adaptive shrinkage function model robust. Experimental results have been evaluated for data compression and the results confirm that the 


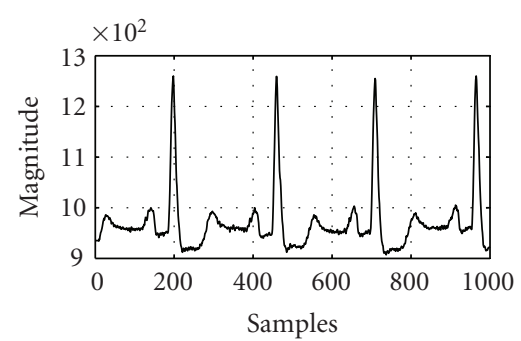

(a)

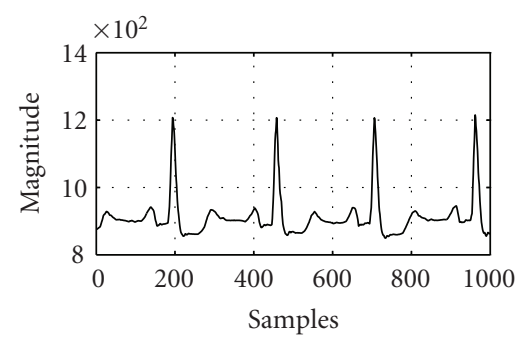

(d)

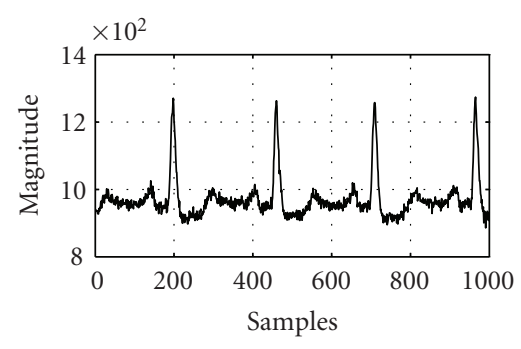

(g)

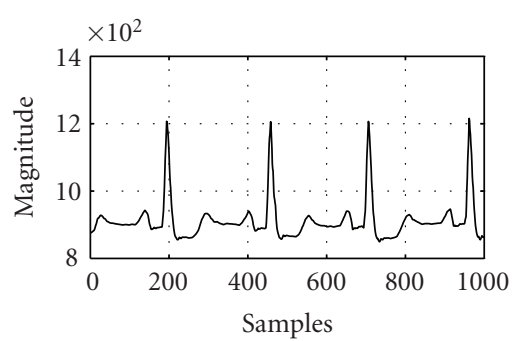

(b)

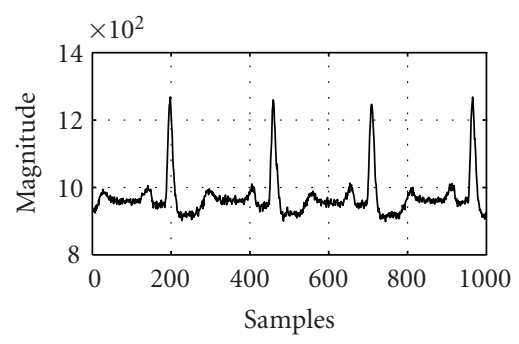

(e)

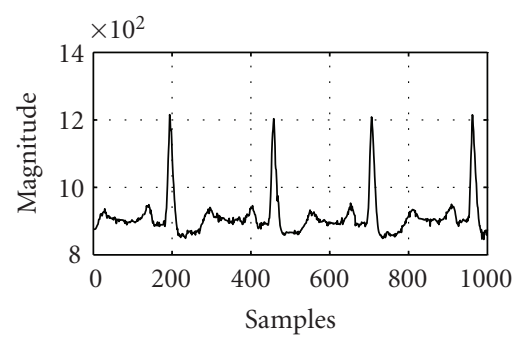

(h)

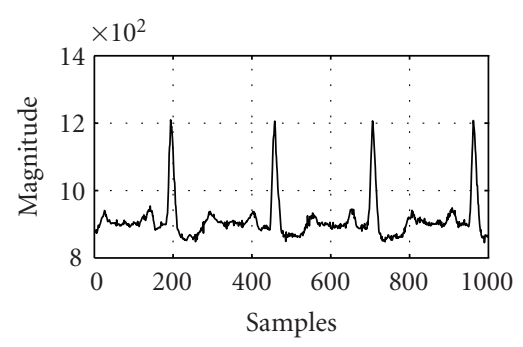

(j)

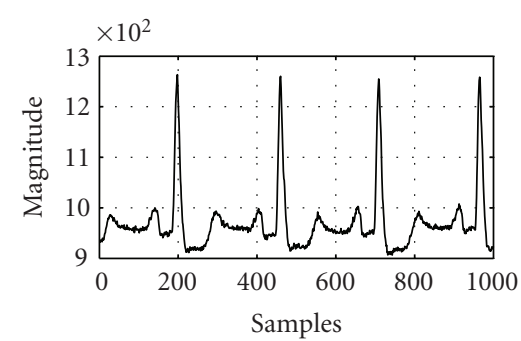

(c)

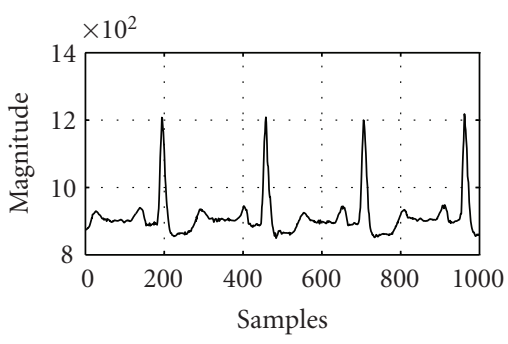

(f)

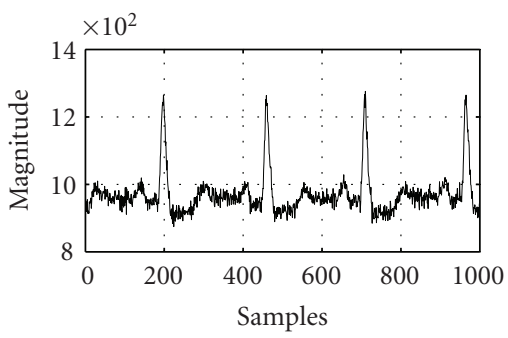

(i)

FIgURE 5: (a) Original ECG signal. (b) Recovered ECG (noise level is $0 \%$ of signal variance). (c) Noisy ECG (noise level is $10 \%$ of signal variance). (d) Recovered ECG (noise level is $10 \%$ of signal variance). (e) Noisy ECG (noise level is $30 \%$ of signal variance). (f) Recovered ECG (noise level is $30 \%$ of signal variance). (g) Noisy ECG (noise level is $50 \%$ of signal variance). (h) Recovered ECG (noise level is $50 \%$ of signal variance). (i) Noisy ECG (noise level is $80 \%$ of signal variance). (j) Recovered ECG (noise level is $80 \%$ of signal variance).

technique is able to achieve good PRD. The subband-adaptive shrinkage function has been further tested with reference to SNR; further, implementation of the new algorithm is simple. The results are visually pleasant and comparable to that of the state-of-the-art algorithms. The proposed subbandadaptive shrinkage model has potential application in denoising signals.

\section{REFERENCES}

[1] D. L. Donoho, "De-noising by soft thresholding," IEEE Transactions on Information Theory, vol. 41, no. 3, pp. 613-627, 1995.

[2] G. A. Bruce and H.-Y. Gao, "Understanding WaveShrink: variance and bias estimation," Biometrika, vol. 83 , no. 4, pp. 727745, 1996. 
[3] C. Stein, "Estimation of the mean of a multivariate normal distribution," Annals of Statistics, vol. 9, no. 6, pp. 1135-1151, 1981.

[4] H.-Y. Gao, "Wavelet shrinkage denoising using the non-negative garrote," Journal of Computational and Graphical Statistics, vol. 7, no. 4, pp. 469-488, 1998.

[5] X.-P. Zhang and M. D. Desai, "Adaptive denoising based on SURE risk," IEEE Signal Processing Letters, vol. 5, no. 10, pp. 265-267, 1998.

[6] H.-Y. Gao and A. G. Bruce, "Waveshrink with firm shrinkage," Statistica Sinica, vol. 7, no. 4, pp. 855-874, 1997.

[7] S. Poornachandra and N. Kumaravel, "Hyper-trim shrinkage for denoising of ECG signal," Digital Signal Processing, vol. 15, no. 3, pp. 317-327, 2005.

S. Poornachandra received his B.E. degree from NIE, Mysore University, and his M.S. degree from MIT, Mangalore University. Currently, he is pursuing research in Anna University in signal processing. He is working as Assistant Professor at the Department of ECE, SSN College of Engineering, Anna University, India. He has authored textbooks in the field of electronics and signal processing. He has presented few papers

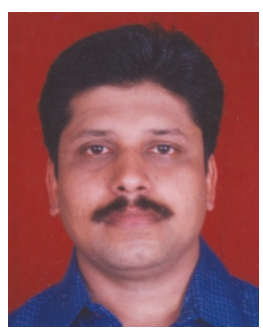
in national and international journals and conferences. His areas of research interest are biosignal processing using wavelet transform based adaptive filter and wavelet shrinkage. He is a life Member of technical bodies like IEEE, ISTE, MIE, and Fellow IETE.

N. Kumaravel received his Ph.D. degree from Anna University in biosignal processing. Currently, he is working as a Professor in the School of ECE, Anna University, Chennai, India. He has published several papers in various national and international journals and conferences. His areas of research interest are noise cancellation techniques using genetic algorithm, neural network, wavelet based adaptive filtering, and

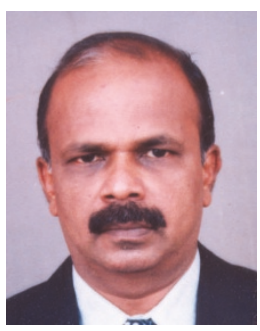
image processing. He is a Member of technical bodies like IEEE, IETE, ISTE, and Biomedical Society of India. 